\title{
Algunas precisiones respecto a los vehículos societarios y los conflictos de interpretación que enfrentamos hoy en día
}

Martín Serkovic Pérez León

Abogado por la Pontificia Universidad Católica del Perú.

SUMARI0:

I. Introducción.

II. Tratamiento legal de las Sucursales.

III. La figura de la Filial.

IV. Los establecimientos permanentes.

V. Algunos conceptos doctrinarios y el Ilamado "Control Empresarial".

VI. Alguna problemática de interpretación actual. 


\title{
RESUMEN:
}

A lo largo de este artículo, se desarrollarán los principales conceptos de las figuras relativas a la vinculación empresarial, haciendo referencia también a aquellos conceptos recogidos de la doctrina y las prácticas comerciales. Dado que las empresas pueden vincularse de muchas maneras, es preciso trazar las principales diferencias entre ellas para evitar incurrir en errores de interpretación, partiendo de la base que dichas figuras comprenden regulaciones legales distintas. También se desarrollará el denominado "control societario" para finalmente analizar cómo abordar los problemas de interpretación que encontramos en la práctica actual.

Palabras clave: Vinculación Empresarial, Control Societario, Sucursal, Filial, Interpretación Normativa.

\begin{abstract}
:
Throughout the following article, the author addresses the main concepts regarding figures related to business engagement, referencing also those concepts addressed in both legal doctrine and business practices. Since companies can be linked in many ways, it is necessary to draw the main differences between them in order to avoid interpretation errors, on the basis that each corporate figure implicates its own regulation. The so-called "corporate control" will also be analyzed in order to determine how to address the interpretation errors found in current practices.
\end{abstract}

Keywords: Business Engagement, Corporate Control, Subsidiary, Branch office, Legal Interpretation.

\section{INTRODUCCIÓN}

Muchas veces se habla indistintamente de las filiales y las sucursales para referirse a aquellas compañías pertenecientes a un mismo grupo empresarial, distintas a la empresa principal o matriz. A lo largo de este artículo, se desarrollarán las principales diferencias entre estas figuras, las cuales presentan hoy en día un problema de interpretación que debe ser tratado.

Durante los últimos años, el Perú ha experimentado un fuerte crecimiento que se ha debido, en gran parte, a las inversiones extranjeras. Cada año, la proyección de las inversiones extranjeras aumenta considerablemente, y las cifras demuestran que dicho crecimiento se ha convertido en una tendencia cada vez más fuerte. La Agencia de Promoción de la Inversión Privada del Perú —en adelante, "Proinversión"ha establecido que en el 2016 se ha alcanzado un Saldo de Inversión Extranjera Directa de US\$ 25,203 millones, a comparación de los US\$ 15,247 del año 2006'. Asimismo, Proinversión ha determinado que los principales inversio- nistas que efectuaron movimientos de capital en el período 2011-2016 fueron empresas de telecomunicaciones, finanzas, minería, energía y petróleo, encontrándose entre los países de origen de dichas empresas Chile, España, Reino Unido y Canadá, entre otros.

Debido a esta creciente proliferación de empresas nacionales y extranjeras que, en la búsqueda de seguir ampliando sus horizontes, deciden establecer otras compañías en el Perú o en el extranjero; surge la necesidad de establecer claramente las definiciones y regulaciones de los vehículos societarios, así como de las formas de relación empresarial o societaria.

De esta manera, comenzaremos por definir y desarrollar cada uno de estos términos para luego pasar a ver algunos otros conceptos que recogemos de la práctica comercial y de la doctrina. Finalmente, analizaremos un ejemplo práctico de interpretación.

Antes de empezar, conviene precisar algunas cuestiones. Cuando nos referimos a la empresa

1. Agencia de la Promoción de la Inversión Privada - PROINVERSIÓN. Consulta: 13 de diciembre de 2017. Disponible en: $<$ http://www.investinperu.pe/modulos/JER/PlantillaStandard.aspx?are=0\&prf=0\&jer $=5652>$. 
principal, también denominada "casa matriz" estamos hablando de aquella compañía que ejerce de alguna manera algún control sobre las demás. Desarrollaremos su concepto más adelante. Como contrapartida, cuando hablamos de una "subsidiaria" simplemente nos referimos a una sociedad que es controlada de alguna manera por otra. Adicionalmente, analizaremos qué entendemos por control societario propiamente dicho.

\section{TRATAMIENTO LEGAL DE LAS SUCURSALES}

De acuerdo al tenor literal del artículo 396 de la Ley 26887 —en adelante, "Ley General de Sociedades" - , se define sucursal como "todo establecimiento secundario a través del cual una sociedad desarrolla, en lugar distinto a su domicilio, determinadas actividades comprendidas dentro de su objeto social"2.

En este sentido, la sucursal no es otra cosa que un establecimiento secundario y permanente que encuentra su razón de ser en la necesidad de que la sociedad principal desarrolle sus actividades en una ubicación distinta a la del domicilio social. En definitiva, podríamos afirmar que la sucursal es considerada por nuestro ordenamiento jurídico como un "brazo" que se desprende de la sociedad principal en cuestión, y que constituye el vehículo para que la sociedad pueda desarrollar sus actividades en otros domicilios. Sobre esta misma línea, la sucursal surge como la necesidad de la dispersión territorial que permite conservar la personería jurídica y la identidad de la sociedad principal; permitiendo que las transacciones entre clientes y proveedores se vuelvan dinámicas y seguras, prescindiendo de la necesidad de recurrir a la creación de una filial ${ }^{3}$.
En cuanto a la personería jurídica, a diferencia de las filiales cuyo concepto desarrollaremos en el siguiente apartado, las sucursales - también llamadas "establecimientos anexos" - , no tienen una actividad distinta a la de la empresa principal. Tanto es así que la sucursal no va a poder desarrollar sus actividades más allá de aquellas expresamente establecidas en el estatuto de la empresa principal. En cierta forma, la sucursal se encuentra limitada, ya que se presenta como una verdadera "extensión" de la empresa a la cual debe su existencia. Además, como consecuencia de las características mencionadas anteriormente, las sucursales cuentan con una relativa independencia de gestión en el sentido de que su dirección y administración se van a circunscribir a las decisiones de los representantes de las empresas de las cuales son "extensión".

Nuestra legislación también establece que la sociedad principal responde por las obligaciones de la sucursal, y tiene sentido, en tanto esta última no puede constituirse como titular de derechos y obligaciones que no sean en nombre de la sociedad a la cual pertenece. En este sentido:

“(...) las obligaciones contraídas a través de la sucursal no dejan ser obligaciones de la sociedad y se encuentran respaldadas por la totalidad del patrimonio de la misma, inclusive por el de la sucursal, con prescindencia del hecho de que tal patrimonio se encuentre asignado exclusivamente a la sucursal o no"4.

Asimismo, el establecimiento de la sucursal implica el nombramiento de un representante legal permanente que tiene las facultades para obligar a la sociedad principal por los actos llevados a cabo por la sucursal. Ello tiene sentido

2. Artículo 396 de la Ley General de Sociedades.

3. Cfr. PÉREZ MONTENEGRO, Kelly. "Tratamiento Tributario Societario de las Sucursales en la Legislación Peruana ¿Convivencia Pacífica o Diferencias Irreconciliables entre ambas Regulaciones? - Primera Parte". En: Derecho y Sociedad, № 33. Lima: 2009, p. 97.

4. ELÍAS LAROZA, Enrique. "Ley General de Sociedades Comentada. Fascículo Primero". Lima: Editora Normas Legales S.A., 1998, p. 805. 
con lo antedicho ya que, al no tener la sucursal personería jurídica propia, todas las obligaciones que contraiga obligarán directamente a la sociedad principal.

Las empresas nacionales pueden constituir sucursales dentro del país como también en el extranjero. A su vez, nuestra legislación establece que las empresas extranjeras pueden constituir sucursales en el Perú. En este último caso, la sociedad constituida en el extranjero deberá acreditar su vigencia y la falta de impedimento para constituir sucursales en la legislación del país donde se constituyó la sociedad principal.

\section{LA FIGURA DE LA FILIAL}

Por otro lado, a diferencia de las sucursales cuya regulación fue desarrollada en el apartado anterior, nuestra Ley General de Sociedades no regula expresamente la figura corporativa de la filial. En este sentido, tampoco encontramos una regulación vigente en el Reglamento de Registro de Sociedades de la Superintendencia Nacional de los Registros Públicos -en adelante, "SUNARP". Sin embargo, la figura de la filial tuvo un tratamiento expreso en la antigua Ley Universitaria —-Ley 23733. Dicha normativa establecía lo siguiente:

"Artículo 5.- (...) Una Universidad no tiene filiales o anexos. Excepcionalmente, puede crear nuevas Facultades, dentro del ámbito departamental, de acuerdo a las necesidades de la región, en concordancia con los planes de desarrollo nacional (...)".

Seguidamente, se promulgó la Ley 27504 que regula la creación de filiales universitarias, estableciéndose la facultad de las universidades de constituir filiales fuera del ámbito departamental de su creación ${ }^{5}$, y derogándose lo establecido en el tercer párrafo del artículo 5 de la antigua Ley Universitaria mencionada anteriormente. Por Ley 28564 , se restituyó la vigencia de este párrafo, quedando nuevamente prohibida la creación de nuevas filiales de universidades públicas y privadas, fuera del ámbito departamental de su sede principal $^{6}$. La Sentencia No 00017-2008-PI-TC emitida por el Tribunal Constitucional declaró inconstitucional el Artículo 2 de la Ley 28564, estableciéndose que no existía una regulación vigente que confiera a la Asamblea Nacional de Rectores —en adelante, "ANR" - o al Consejo Nacional para la Autorización y Funcionamiento de Universidades -en adelante, "CONAFU" - competencias para autorizar el funcionamiento de filiales.

Asimismo, el Reglamento de Funcionamiento de Filiales Universitarias definía a la filial como "la unidad académica desconcentrada, ubicada fuera del departamento en que se creó la universidad, que ofrece carreras profesionales o estudios de post grado".

Cabe agregar que la Ley 30220 —en adelante, "la nueva Ley Universitaria" - establece que entre las funciones de la SUNEDU se encuentra la de aprobar o denegar las solicitudes de licenciamiento de universidades, filiales, facultades, escuelas y programas de estudios conducentes a grado académico, y normar y supervisar las condiciones básicas de calidad exigibles para el funcionamiento de las universidades, filiales, facultades, escuelas y programas de estudios conducentes a grado académico, así como revisarlas y mejorarlas periódicamente. En este sentido, no aclara nada más con respecto a la creación de filiales.

De manera que, en nuestro ordenamiento legal, las filiales no han recibido mayor tratamiento a nivel regulatorio. Ahora bien, cabe resaltar brevemente lo establecido por la doctrina en general respecto a la regulación societaria de la filial.

5. Cfr. artículo 1 de la Ley 27504 que regula la creación de filiales universitarias y otorga facultades adicionales a la Asamblea Nacional de Rectores -ANR-.

6. Cfr. artículo 2 de la Ley 28564. 
La filial es una empresa subsidiaria independiente de la empresa o casa matriz pero que es controlada por esta. Ahora bien, a pesar de que la matriz ejerce control sobre la filial, esta última tiene personería jurídica propia: cuenta con una organización, un domicilio y un patrimonio propio, a diferencia de las sucursales.

La independencia de las filiales respecto a la casa matriz, va a verse siempre condicionada de alguna manera por el control ejercido por esta última en virtud de ostentar la mayoría del paquete accionario. A diferencia de la sucursal, al tener personería jurídica propia, la filial responderá por las obligaciones que contraiga sin que ello implique a la sociedad principal o casa matriz.

Podríamos asimilar la figura de la filial con cualquier subsidiaria, es decir, cualquier sociedad - adquiera la figura societaria que sea-que es controlada por otra en virtud de ostentar esta última la mayoría del paquete accionario, o el control en el órgano de administración, entre otras posibilidades.

Ahora bien, como podemos ver de los conceptos analizados, las sucursales y filiales se presentan como figuras totalmente distintas. Mientras que la sucursal se presenta como una extensión de la sociedad principal creada para que esta última pueda funcionar en domicilios diferentes, la filial es una entidad con personería jurídica propia que es totalmente independiente de la casa matriz. Solo a efectos comparativos - ya que nuestra normativa societaria sólo regula el establecimiento de sucursales- podríamos decir que la constitución de una u otra condicionaría el tipo de vinculación entre la sociedad principal y la filial o sucursal, por las consideraciones que se han expuesto anteriormente. En este sentido:

“(...) si se desea que la vinculación sea permanente en el tiempo y con plena responsabilidad de los actos por parte de la empresa matriz, lo recomendable sería optar por crear una sucursal; por el contrario, si la vinculación entre matriz y subsidiaria no tiene una vocación de permanencia en el tiempo, ni se desea que los actos de la subsidiaria involucren el patrimonio de la empresa principal, lo recomendable sería optar por constituir una filial, o por adquirir acciones de una empresa de modo tal que su porcentaje de participación en el accionariado le asegure pleno mando sobre esta empresa"7.

\section{LOS ESTABLECIMIENTOS PERMANENTES}

En nuestro país el establecimiento permanente es regulado a efectos tributarios. Al respecto, el artículo 3 del Reglamento de la Ley del Impuesto a la Renta establece que constituye establecimiento permanente:

"(...) Cualquier lugar fijo de negocios en el que se desarrolle total o parcialmente, la actividad de una empresa unipersonal, sociedad o entidad de cualquier naturaleza constituida en el exterior. En tanto se desarrolle la actividad con arreglo a lo dispuesto en el párrafo anterior, constituyen establecimientos permanentes los centros administrativos, las oficinas, las fábricas, los talleres, los lugares de extracción de recursos naturales y cualquier instalación o estructura, fija o móvil, utilizada para la exploración o explotación de recursos naturales; 2) Cuando una persona actúa en el país a nombre de una empresa unipersonal, sociedad o entidad de cualquier naturaleza constituida en el exterior, si dicha persona tiene, y habitualmente ejerce en el país, poderes para concertar contratos en nombre de las mismas; y 3) Cuando la persona que actúa a nombre de una empresa unipersonal, sociedad o entidad de cualquier naturaleza constituida en el exterior, mantiene habitualmente en el país existencias de bienes o mercancías para ser negociadas en el país por cuenta de las mismas".

7. IRIARTE \& ASOCIADOS. "Sucursales y Filiales: Diferencias y Similitudes en la Práctica y Ordenamiento Legal". En: E-boletín legal de Derecho Corporativo. Lima: 2013, Año III, N 13. Disponible en: < http://www.iriartelaw.com/ sites/default/files/boletin-co-13.pdf>. 
El objeto de la regulación del establecimiento permanente es el de gravar la renta generada en el Perú por los sujetos no domiciliados. Nuestra legislación tributaria establece que los sujetos considerados domiciliados tributarán por sus rentas de fuente mundial, y los no domiciliados, lo harán por sus rentas de fuente peruana ${ }^{8}$. Ahora bien, como mencioné anteriormente, esto aplica solo a efectos tributarios: nuestra Ley General de Sociedades no define qué es el establecimiento permanente.

\section{ALGUNOS CONCEPTOS DOCTRINARIOS Y EL LLAMADO “CONTROL EMPRESARIAL”}

Tal como mencionamos en la primera parte de este artículo, trataremos brevemente algunos conceptos que se suelen utilizar en la práctica cotidiana comercial y en la doctrina.

Se suele denominar a la empresa principal como "casa matriz". Tomamos esa acepción como un concepto doctrinario para referirnos a aquella sociedad principal -ello, en relación a las sucursales o filiales con las que cuente- respecto de la cual "dependen", de alguna manera, una o más sociedades.

Cuando hacemos referencia a la relación controlada-controlante, nos referimos a la existente entre la casa matriz y las otras sociedades que se encuentran controladas por esta, ya que la primera ostenta la mayoría del paquete accionario. Nuevamente, nuestra Ley General de Sociedades no regula el concepto de "casa matriz", sino que es un concepto que generalmente utilizamos en la práctica comercial y que recogemos de la doctrina. La doctrina extranjera también hace mención a la "sociedad holding" que es aquella sociedad matriz que tiene un control sobre las otras sociedades en tanto posee la mayor parte del paquete accionario. Muchas veces la sociedad holding es simplemente poseedora de las acciones de las otras sociedades, pero no desarrolla actividades en sí misma, sino a través de sus sociedades controladas. En definitiva, se crea estas sociedades holding a efectos de limitar la responsabilidad patrimonial, también la incorporación de socios para esas actividades, la necesidad de descentralización y la distinta localización geográfica9

Esto nos lleva a preguntarnos cuándo estamos frente al llamado"control societario o empresarial". Si bien nuestra Ley General de Sociedades solo habla de la regulación de las sucursales, podemos encontrar una definición clara de "control" en el Reglamento de Propiedad Indirecta, Vinculación, y Grupos Económicos - RSMV N ${ }^{\circ}$ 019-2015-SMV/01. En este sentido, se define al control como "la influencia preponderante y continua en la toma de decisiones de los órganos de gobierno de una persona jurídica u órganos que cumplan la misma finalidad en el caso de un ente jurídico"10. En definitiva, tal como se estableció anteriormente respecto a la sociedad holding, el control va a estar relacionado siempre con la tenencia de una parte relevante del paquete accionario de la otra sociedad.

Por otro lado, el RSMV N 019-2015-SMV/01 también establece lo que es el grupo económico, tomando como principal requisito el control de una entidad o de una o varias personas naturales sobre las demás entidades, siendo necesario para conformar el grupo económico que haya al menos dos entidades.

\section{ALGUNA PROBLEMÁTICA DE INTERPRETACIÓN ACTUAL}

Pese a que los conceptos de las formas de vinculación societaria desarrolladas en los apartados anteriores parecen, en un principio, claros y totalmente diferenciables, en la práctica han surgido algunos inconvenientes de interpretación.

8. Cfr. artículo 6 de la Ley del Impuesto a la Renta.

9. FAVIER DUBOIS, Eduardo (padre e hijo). "La Sociedad "Holding" y la Sociedad "Filial" en el Derecho Argentino". Errepar. DSE. № 276, Tomo XXII, 2010. p. 1189.

10. Artículo 6 del Reglamento de Propiedad Indirecta, Vinculación, y Grupos Económicos RSMV Nº19-2015-SMV/01. 
Como ejemplo, tomaremos un caso de interpretación por parte de la Superintendencia Nacional de Educación Superior Universitaria -en adelante, "SUNEDU"-. Dicha entidad, haciendo una interpretación errada respecto a los conceptos referidos, envió un oficio a una universidad privada indicando que la misma no habría cumplido con registrar ante la SUNEDU una supuesta filial que se encuentra en una ciudad en el interior del país.

Lo cierto es que esta supuesta "filial" no era una persona jurídica independiente — no era una filial — sino que se trataba, más bien, de una sucursal y, por ende, no se encontraba sujeta a los requisitos de registro especificados por la SUNEDU. En efecto, había sido constituida con la finalidad de que funcione como una extensión de la sociedad principal. Asimismo, se había acordado que la sucursal se iba a dedicar exclusivamente a la prestación de servicios educativos, especialmente de educación superior universitaria. No iba a otorgar títulos por sí misma, ni a desarrollar una currícula de estudios de manera independiente por sí misma. Se trataba simplemente de extender las facilidades de la universidad a ciertas ciudades, para que las aulas de esta se extiendan al territorio de estas ciudades.

Es decir, todas las actividades a ser prestadas por la sucursal eran exclusivamente la extensión de las que prestaba la sociedad —la universidad-. En este sentido, bajo ningún concepto se podía interpretar que esta entidad se constituiría como una entidad con personalidad jurídica propia ya que efectivamente prestaría los mismos servicios que la sociedad principal y que funcionaría efectivamente como una extensión de la misma.

Bien sabemos que las filiales pueden cumplir determinado objeto social relacionado a la casa matriz, como que pueden no hacerlo, ya que tienen personería jurídica propia. En cambio, las sucursales, como mencionamos anteriormente, solamente pueden llevar a cabo aquellas actividades que disponga la dirección de la sociedad principal. Es decir, pueden llevar a cabo las mismas actividades o aquellas que la sociedad principal determine, pero nunca fuera de las previstas en el estatuto.

Ahora bien, la interpretación efectuada por la SUNEDU suponía que la universidad privada había incumplido con registrar ante la SUNEDU la supuesta filial - se consideró a la sucursal como "filial" - sino que se imputó que, además, se estaba incumpliendo con la Ley 29971 del año 2012, que estableció la moratoria de creación de universidades públicas y privadas, prohibiéndose también la constitución de filiales. Una vez más, la autoridad interpretó que, bajo el concepto de "filial", estaban comprendidas las sucursales.

La autoridad llegó a esta interpretación sobre la base de una ley muy clara y con mención taxativa y expresa sobre a quien se aplicaba la suspensión o moratoria. La ley señaló que la suspensión aplicaba a las universidades y filiales. Siendo el caso que no cabe distinguir allí donde la ley no distingue, es clarísimo que los alcances de la norma no podían vía interpretación de autoridad alguna extenderse a las sucursales; sin embargo, ello sucedió.

Estas confusiones de interpretación, lamentablemente, se dan debido a que, en nuestra legislación societaria, no están regulados términos o figuras que luego son utilizados en normas específicas en virtud de tratarse de conceptos desarrollados por la doctrina comparada. Al no estar expresamente regulados se pretende hacer interpretaciones extensivas para lograr el fin que se persigue, sin tener en consideración que con ello se trasgrede el ordenamiento jurídico, la seguridad jurídica y el estado de derecho.

Lo lamentable es que estos errores de interpretación o interpretaciones forzadas, generan en la gran mayoría de los casos, consecuencias jurídicas y económicas serias que perjudican a los agentes económicos. 